\title{
Prevalence of dry eye amongst black and Indian university students aged $\mathbf{1 8}-\mathbf{3 0}$ years
}

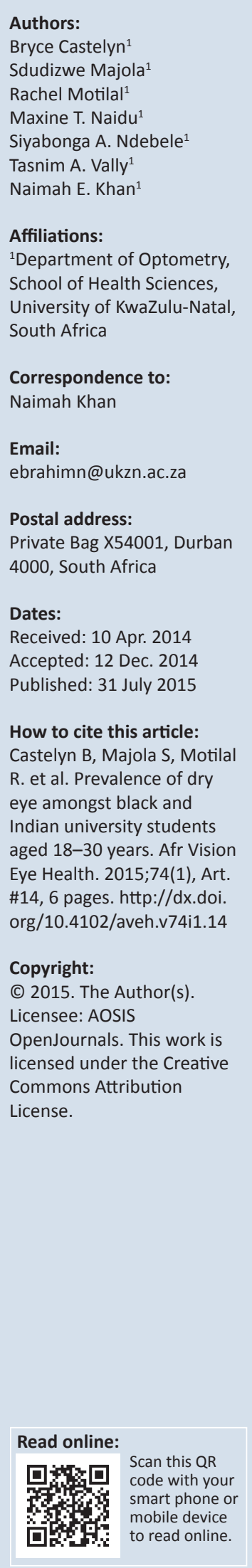

Background: The overall prevalence of dry eye in South Africa seems to be increasing. University students work under conditions predisposing them to dry eye, which may affect some tasks. The predominant race groups at the University of KwaZulu-Natal (UKZN) are black and Indian, which suggested a study in this student population to assist the diagnosis and management of such subjects.

Aim and setting: To compare the prevalence of dry eye amongst black and Indian students at the UKZN's Westville campus.

Methods: One hundred participants, equally divided by gender and race, between 18 and 30 years old were enrolled. Dry eye symptoms were investigated by the ocular surface disease index (OSDI), tear thinning time (TTT), tear breakup time (TBUT) and Schirmer's 2 in that sequence on both eyes of each participant.

Results: The OSDI revealed that $41 \%$ of participants had some dry eye symptoms whilst $59 \%$ had no symptoms. Clinical testing showed that $81 \%$ of participants had dry eye. Half of the black participants had dry eye symptoms and $82 \%$ had clinical signs of dry eye. Of the 50 Indian participants, $32 \%$ had dry eye symptoms and $80 \%$ had clinical signs. Of the 50 male participants, $34 \%$ were symptomatic and $86 \%$ had clinical signs. Of the 50 female participants, $48 \%$ had dry eye symptoms and $76 \%$ had clinical signs. Participants were asymptomatic even in the presence of clinical dry eye signs.

Conclusion: For both races and genders, clinical signs of dry eye were more common than symptoms. Black participants were more likely to report symptoms than Indians, and more women than men reported having symptoms. Male participants were more likely than female to have clinical signs of dry eye.

\section{Introduction}

The outer portion of the eye comprises a number of structures, each with a specific function. The ocular surface, tear film, lacrimal glands and eyelids act as a functional unit to preserve the quality of the refractive surface of the eye, to resist injury, and to protect the eye against changing bodily and environmental conditions. ${ }^{1}$ The tear film plays a vital role in nourishing, lubricating and protecting the ocular surface. ${ }^{2}$

Dry eye is often a consequence of tear film anomaly. There are three distinct layers of the tear film: ${ }^{3}$ an outer lipid layer, a watery aqueous layer in the middle, and a slimy mucous layer which lies in apposition to the cornea. A deficiency in any one of these layers could result in the subject experiencing discomfort and exhibiting signs of dry eye. Furthermore, tears pass through four processes: production by the lacrimal gland, distribution by blinking, evaporation from the ocular surface, and drainage through the naso-lacrimal duct. Abnormalities in any one of these steps can also lead to dry eye. ${ }^{4}$

Dry eye can be divided into two subgroups: tear deficient dry eye (TDDE) and evaporative dry eye (EDE). ${ }^{5}$ A deficiency in the lipid layer of the tear film is aetiological in about $80 \%$ of patients suffering from dry eye, which results in excessive evaporation. ${ }^{6}$ Dry eye conditions can also be classified into those with adequate aqueous tear production and those with aqueous tear deficiency. Adequate aqueous dry eye patients suffer from meibomian gland dysfunction that can result in lipid tear deficiency. Aqueous tear deficiency may be subclassified into non-Sjogren's and Sjogren's syndrome. Patients with non-Sjogren's aqueous tear deficiency have less severe symptoms and ocular surface disease than those with Sjogren's syndrome. In Sjogren's syndrome, immune-mediated destruction of the lacrimal gland results in severe aqueous tear deficiency. Aqueous tear deficiencies lead to an ocular surface disease termed keratoconjunctivitis sicca (KCS). KCS results from abnormal terminal differentiation of the ocular surface epithelia and 
is associated with marked reduction in mucin production by these cells. ${ }^{7}$ Dry eye can lead to damage of the ocular surface as well as result in symptoms of ocular discomfort and visual disturbance ${ }^{8}$ such as blurred vision or a burning/ gritty feeling of the eyes. Although dry eye presents symptomatically as a rather common ocular condition, it tends to be difficult to clinically diagnose as there is little correlation between the symptoms with which the patient presents and objective signs of ocular compromise. Dry eye is most commonly a source of irritation and discomfort to the affected individual and, in more severe cases, it can result in a marked predisposition to certain eye diseases owing to impairment of the protective ability of the tear film. Dry eye has a negative impact on the quality of life of affected individuals ${ }^{9}$ and can affect their ability to conduct everyday tasks. $^{10}$

Many factors are considered to influence dry eye symptoms and disease, some of which are: diurnal variation in tear volume, contact lens wear, age, systemic diseases, gender, diet, water intake and environmental factors such as air conditioning. The diagnosis of dry eye requires a battery of interviews and tests to be performed such as dry eye questionnaires and clinical testing. These include symptom assessment, contact lens and medical history, slit-lamp biomicroscopic evaluation of the eyelids, evaluation of the meibomian glands, assessment of tear film quality, tear meniscus height, assessment of blink quality, fluorescein tear break up time (TBUT), fluorescein and rose Bengal staining of the cornea and conjunctiva, phenol red thread test and the Schirmer tests. ${ }^{11}$

Questionnaires, many of which have also been used as screening tools, have been an integral tool in dry eye diagnosis for many years. Questionnaires based on symptoms have been used to screen populations for undiagnosed cases as well as prevalence. Some commonly used questionnaires include the McMonnies questionnaire, the dry eye syndrome questionnaire (DES), the contact lens dry eye questionnaire (CLDEQ), the ocular surface disease index (OSDI) and the Schaumberg questionnaire.

The OSDI questionnaire was used in the present study; it is a 12-item screening questionnaire for dry eye. Three subscales sequentially probe for symptoms of ocular irritation, the impact of vision-related functioning, and environmental triggers of dry eye syndrome. The questionnaire is criticised because the step in difficulty between each category is not constant and the difficulty of all questions is not necessarily comparable. ${ }^{12}$ In this questionnaire, there has been an attempt to segregate subjective symptoms based on the severity of dry eye. ${ }^{13}$ Pult et al. ${ }^{14}$ found that the OSDI can assist in the prediction of dry eye. The strength of this questionnaire is that it probes for three different aspects.

A number of clinical tests can be used to diagnose dry eye, but the two commonly used tear tests are the Schirmer test and TBUT which were used in this study. The tear thinning time test (TTT) was also performed in this study. TTT is widely used because of its non-invasive nature and ease of performance.

The Schirmer test is considered one of the most useful in detecting the severest, most tear deficient dry eye. ${ }^{15}$ It was first described by Schirmer in 1903 and is still the method most commonly used clinically to evaluate aqueous tear production. ${ }^{16,17}$ Traditionally, the procedure for the Schirmer test involves placing a Schirmer test strip hooked onto the inferior tarsus while the patient blinks normally. After five minutes, the strip is removed and the wet length measured with a millimetre rule. The test can be performed without the administration of a topical anaesthetic (Schirmer 1), where both the basal and reflex secretion of the tears is measured. Alternatively, the Schirmer 2 test can be performed by instilling a local anaesthetic before insertion of the Schirmer strip, and thus only the basal secretion rate will be tested. A wet portion of the strip less than $5 \mathrm{~mm}$ is considered abnormally low. ${ }^{4}$

The TBUT is used mainly to assess the stability of tears. ${ }^{15}$ Tear stability describes the effectiveness of the cohesive forces present between the three layers of the tear film. When one or more of the layers break up, the tear film will be unstable. TBUT is the time interval between a complete blink and the first appearance of a dry spot in the precorneal tear film after the installation of fluorescein viewed with a cobalt blue filter. ${ }^{18,19}$ Traditionally, three readings are taken and the results averaged. This technique has gained worldwide popularity as it is simple and convenient to perform. ${ }^{18}$ Even though there is a wide range of values amongst individuals, there is a general agreement that a TBUT shorter than 10 seconds reflects tear film instability, and a TBUT shorter than 5 seconds is a marker indicative of dry eye.

Tear thinning time test is a test of tear integrity, and commonly referred to as non-invasive break up time (NIBUT). It is the time interval in seconds between the blink and the first observable distortion of the reflected mire from a keratometer. Some practitioners view this method to investigate tear film stability as more accurate owing to its non-invasive nature. However, Cho and Douthwaite $^{20}$ have reported that NIBUT values seem to be significantly higher than those of TBUT. The limitation of the former test is that only a small area of the pre-ocular surface is tested.

The overall prevalence of dry eye in South Africa seems to be increasing, as a number of South African practitioners report seeing more dry eye patients. Gillan ${ }^{21}$ used the OSDI to ascertain the prevalence of dry eye symptoms in a research population based in Johannesburg, South Africa. The results of the study yielded a prevalence of $64 \%$; this is a relatively high value compared with that reported by other studies $^{11,22}$ where the prevalence varied between $0.39 \%$ and $33.7 \%$. University students often work under conditions predisposing them to dry eye (which may impede them from carrying out required tasks). UKZN has a rich history of the 
merging of many cultures, with the predominant race groups at the University being black and Indian, which prompted the need to perform a prevalence study in this population among students of this age group. The results of the study will assist practitioners in the diagnosis and management of such patients.

\section{Materials and methods}

The study was approved by The Research and Ethics Committee, Faculty of Health Sciences, UKZN, South Africa, and all ethical guidelines were adhered to during and after the study. Sample size was determined after the conduction of a pilot study as well as consultation with a UKZN statistician. A randomised convenient sampling method was used to select the 100 participants who took part in the study. An equal number of male and female as well as black and Indian participants were included. Only participants aged 18-30 years were included as the study focused on university students, and this is the common age range of UKZN students. The black and Indian racial groups were chosen as they represent the majority of the population in the study location (UKZN, Westville Campus). Following written informed consent from each participant, screening questions were asked regarding the use of contra-indicated medications as well as whether the female participants were currently on their menstrual cycle. The OSDI questionnaire was then administered and thereafter a brief slit lamp examination was performed as a preliminary test to rule out any ocular surface pathology (for example, hordeolum or chalazion) that might contribute towards dry eye. Participants who passed this screening then had the TTT, TBUT and Schirmer's 2 tests conducted on both eyes in that sequence. Normal clinical test values used were greater than 12 seconds for TTT, greater than 10 seconds for TBUT and greater than $10 \mathrm{~mm}$ for Schirmer's 2.

Each clinical test was conducted by one researcher for the duration of the study. In an effort to eliminate bias, the results from the preceding clinical tests were not divulged to the individual researchers during the conducting of their specified test. For TTT and TBUT, stopwatches were used by the examiners to ensure accuracy of timing. The endpoint for TTT was the first appearance of distorted mires. The endpoint for TBUT was the first appearance of a dark spot amongst the fluorescein. To allow for the participant's ocular surface to return to its habitual state (draining of fluorescein and saline), a time interval of three minutes between tests was observed. Each testing station had its own separate recording sheet on which the results for that specific test were recorded. After each data collection session, the test results were combined onto a single record sheet for each participant. Data were analysed by means of descriptive statistics and the Statistical Package for Social Sciences (SPSS) version 21.

\section{Results}

Each participant was tested for the presence or absence of dry eye. Moisture readings were obtained for the left and right
TABLE 1: Ocular surface disease index results for 100 young participants.

\begin{tabular}{ll}
\hline Severity of dry eye & Number of participants \\
\hline Normal & 59 \\
Normal to mild & 7 \\
Mild & 3 \\
Mild to moderate & 17 \\
Moderate & 13 \\
Moderate to severe & 1 \\
Severe & 0 \\
\hline Total number of participants & $\mathbf{1 0 0}$ \\
\hline
\end{tabular}

eye of each student by using the TTT, TBUT and Schirmer 2 tests. The following readings resulted in a student being classified as having dry eye: TTT less than $12 \mathrm{~s}$ on at least one eye, TBUT less than $10 \mathrm{~s}$ and Schirmer's 2 result $<10 \mathrm{~mm}$ on at least one eye. The purpose of the analysis was to determine whether age, race (black, Indian) or gender was related to the prevalence of dry eye (Table 1).

Results of the OSDI questionnaire prior to clinical testing showed that $41 \%(41 / 100)$ of all participants were symptomatic for dry eye. Across races, it was found that $50 \%$ $(25 / 50)$ of black participants were symptomatic for dry eye whilst 32\% (16/50) of Indian participants were symptomatic. Accordingly, black participants were more likely to report dry eye symptoms than Indian participants. Forty-eight per cent $(12 / 25)$ of black men, 52\% (13/25) of black women, $20 \%$ $(5 / 25)$ of Indian men and $44 \%(11 / 25)$ of Indian women were found to have symptoms of dry eye.

Objective clinical testing (TTT, TBUT and Schirmer's 2) diagnosed $81 \%(81 / 100)$ of all participants as having dry eye. Figure 1 shows that $86 \%(43 / 50)$ of male participants and $76 \%$ $(38 / 50)$ of female participants had signs of dry eye. Logistic regression with TTT classifications and age, race and gender as explanatory variables revealed no significant effects. However, logistic regression with TBUT classifications and age, race and gender as explanatory variables revealed that the effect on age was statistically significant $(p=0.02)$. Means and standard deviations for age for the normal and dry eye TBUT groups were compared and a significant difference between the groups was found ( $p=0.032$ ). The age for the dry eye group was significantly greater than that of the normal eye group. Logistic regression with Schirmer 2 classifications and age, race and gender as explanatory variables revealed that race and gender had significant effects $(p=0.02$ and $p=$ 0.04 respectively).

Considering the Schirmer 2 test results only, the prevalence of dry eye is greater amongst black than Indian students. The odds ratio was:

$\frac{27 \times 33}{17 \times 23}=2.28$

[Eqn 1]

Therefore, black students are slightly more than twice as likely to have dry eye than Indian students. Black male students are more likely to have dry eye than Indian male students, whilst the prevalence of dry eye is the same for female students. 


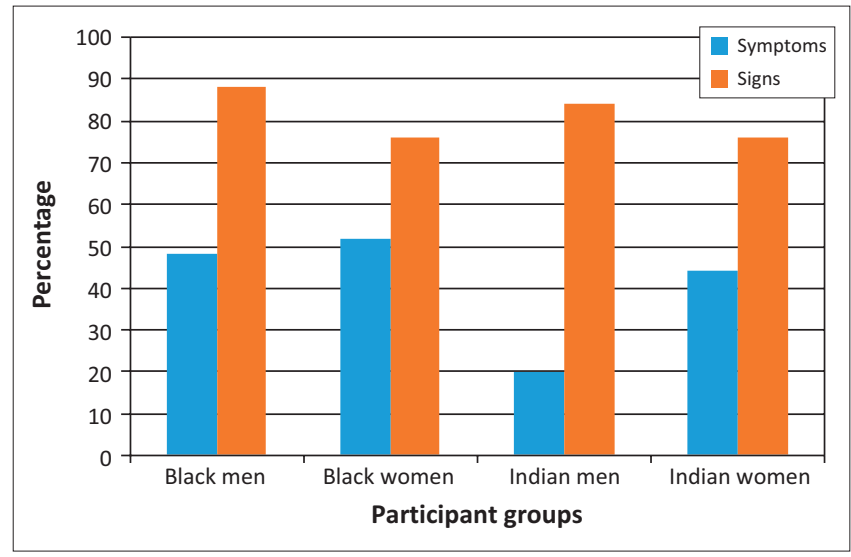

FIGURE 1: Prevalence of symptoms and signs amongst participant groups ( $N=$ 25 for each group).

\section{Discussion}

The debate around reported symptoms versus clinical signs has been ongoing but most practitioners have concluded that both symptoms and clinical signs are important predictors for dry eye disease. ${ }^{2}$ Questionnaires based on symptoms have been used to screen populations for undiagnosed cases as well as prevalence. Dry eye symptom questionnaires are also useful tools in drug trials and in assessing the response to dry eye therapy. Simpson et al. ${ }^{23}$ assessed dry eye symptoms using four questionnaires and found that the results were highly correlated. The present study revealed that women were more likely than men to report dry eye symptoms, which is in agreement with the findings of Lekhanont et al. ${ }^{24}$ A possible reason for this probability could be that women's sensitivity threshold is less than that of men or that women are more aware of their bodily responses.

Across races, the present study revealed that 50\% (25/50) of black participants were symptomatic for dry eye whilst $32 \%$ $(16 / 50)$ of Indian participants were found to be symptomatic. Consequently, black participants were more likely than Indian to report dry eye symptoms.

Results of the OSDI questionnaire prior to clinical testing revealed that $41 \%(41 / 100)$ of all participants were symptomatic for dry eye. The prevalence of dry eye using objective testing methods (TTT, TBUT and Schirmer's 2) diagnosed $81 \%(81 / 100)$ of all participants as having dry eye. Dry eye prevalence is being investigated by researchers in many countries. Gillan ${ }^{21}$ used the OSDI to ascertain the prevalence of dry eye symptoms in a research population based in Johannesburg, South Africa. The results of the study yielded a prevalence of $64 \%$; this is a relatively high value compared with that reported by other studies ${ }^{11,22}$ where the prevalence varied between $0.39 \%$ and $33.7 \%$. Dry eye syndrome in elderly Tibetans at high altitude was investigated by Lu et al. in $2008 .{ }^{25}$ A total of 1840 participants were tested, of whom $52.4 \%$ were symptomatic. A statistically significant correlation between dry eye symptoms and signs was present. Guo et al. ${ }^{26}$ investigated dry eye symptoms amongst elderly Mongolians at high altitude; 1816 participants were tested and $50.1 \%$ were symptomatic. A statistically

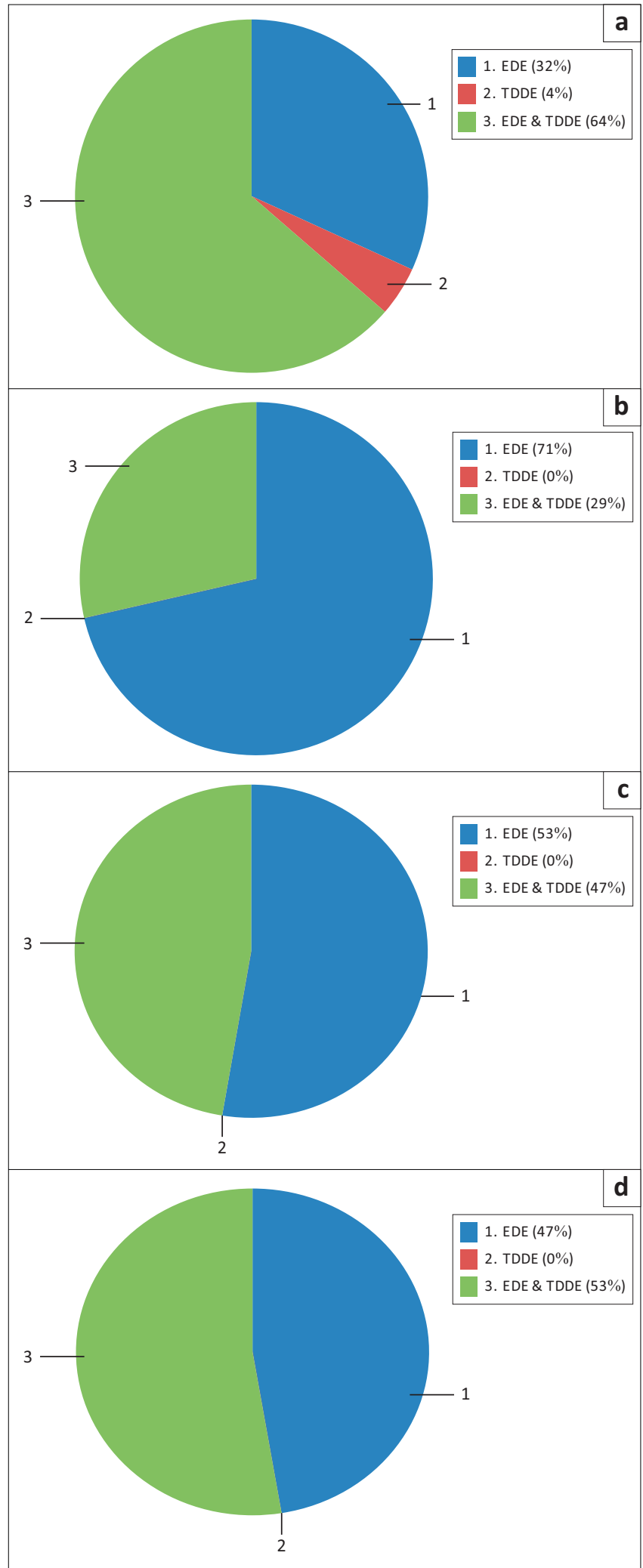

FIGURE 2: Prevalence of dry eye sub-classes amongst (a) 25 black men, (b) 25 Indian men, (c) 25 black women and (d) 25 Indian women.

significant correlation between dry eye symptoms and signs was present. The Beijing Eye Study ${ }^{27}$ was conducted in 2001 and comprised 4439 participants. A random sample of 1957 of the participants was used to assess dry eye symptoms. The subjective symptoms of dry eye were positive in $21 \%$ of the participants. A population-based study of dry eye symptoms 
in Indonesia yielded a prevalence of $27.5 \%{ }^{28}$ In contrast to other studies, dry eye symptoms were more prevalent in men. Brewitt and Sistani ${ }^{29}$ stated that one in four patients consulting an ophthalmologist in Germany complained of symptoms of dry eye. After considering all these effects, the results from Gillan ${ }^{21}$ are most comparable and similar to the present study as the former study was conducted in South Africa.

Eighty-six per cent $(43 / 50)$ of male participants and $76 \%$ $(38 / 50)$ of female participants had signs of dry eye. Therefore, male subjects are more likely to have dry eye than female subjects, indicating that male gender is a predisposing factor to dry eye. These findings agree with those of a population-based study of dry eye symptoms in Indonesia ${ }^{28}$ where the prevalence of dry eye was reported as 1.4 times higher amongst men than women. Women, however, have generally been recognised as having a preponderance of dry eye symptoms. ${ }^{30,31}$ Ozdemir and Temizdemir ${ }^{32}$ reported no significant difference in dry eye symptoms between the sexes. Patel and Farrell ${ }^{33}$ also reported no significant differences in tear film stability between men and women. Possible reasons for dry eye being more prevalent in men than women may stem from greater awareness amongst women than men of health maintenance. Differences in dietary intake and nutritional supplements might also have influenced this prevalence, and possible lifestyle differences between men and women could affect predisposition to dry eye.

Black men showed the highest prevalence of dry eye, at $88 \%$, which could be because of differences in anatomical structure of the eyelid between the two races: black people have, on average, a larger palpebral aperture than Indian people, which exposes a larger area of their ocular surface and subsequently increases the risk of dry eye occurring. Of the black male participants, $64 \%(14 / 22)$ were classified as having a combination of EDE and TDDE, 32\% (7/22) were classified as having solely EDE, and 4\% (1/22) as having solely TDDE. Amongst the Indian male participants, $84 \%$ $(21 / 25)$ were found to have some form of dry eye, of whom $29 \%(6 / 21)$ were classified as having a combination of EDE and TDDE, and $71 \%(15 / 21)$ as having solely EDE, whilst none had solely TDDE. It was found that Indian men were more predisposed to having EDE between the two genders and race groups.

The majority of female participants tested positive for dry eye but little variation was noted between the two race groups. It was found that $76 \%(19 / 25)$ of both black and Indian female participants presented with some form of dry eye. Of the black female participants found to have dry eye, $47 \%(9 / 19)$ were classified as having a combination of EDE and TDDE, 53\% (10/19) were classified as having solely EDE and none were found as having solely TDDE. Amongst the Indian female participants found to have dry eye, 53\% (10/19) were classified as having a combination of EDE and TDDE, 47\% (9/19) were classified as having solely EDE and none was found to have solely TDDE. The high prevalence of dry eye found when using objective test values as diagnostic criteria could be attributed to the study population being more predisposed to risk factors of dry eye such as working in air-conditioned environments as well as undertaking long-duration, close-up tasks (e.g. studying). Additionally, the 'normal' values used for the diagnosis of dry eye were based on international norms that may not be applicable to the South African population; this could have resulted in an over-estimation of dry eye prevalence on objective testing.

It was found that $39 \%$ of all participants had a combination of EDE and TDDE. Forty-one percent of participants had solely EDE, and 1\% had solely TDDE, indicating that more people could suffer from EDE than TDDE. This conclusion correlates with a study by Albietz ${ }^{34}$ which reported a higher prevalence of EDE than TDDE. The effect of age was significant for TBUT results, which indicated that the older the participant was, the lower the TBUT result. The Schirmer test revealed significant effects for both race and gender. Dry eye was more prevalent amongst blacks than Indians, and black men were more likely than Indian men to have dry eye.

The above results show a discrepancy between symptoms and objective results, with participants across all four groups being less likely to complain of dry eye symptoms despite signs being present. This finding contradicts a study conducted by Schein et al. ${ }^{35}$ (that dry eye could be diagnosed solely on the presence of symptoms) but does, however, concur with the findings by Pflugfelder et al. ${ }^{7}$ who concluded that symptoms alone are inadequate for the diagnosis of dry eye. This lack of correlation between symptoms and signs could be attributed to the subjective responses of participants when reporting symptoms as opposed to the objectiveness imposed by clinical testing.

\section{Conclusion and recommendations}

The present study found a lack of correlation between the symptoms and signs of dry eye. The prevalence of dry eye was found to be greater when objective testing was used and lower when symptoms only were used as diagnostic criteria. Female participants were found to be more likely than male to report symptoms of dry eye, whilst it was found that men were more likely than women to present with signs. Black participants were found to be more likely than Indian participants to report symptoms of dry eye but the prevalences of signs of dry eye were similar between the two races. Overall, it was found that black men had the highest prevalence of dry eye amongst our study population; this has implications for the practising clinician in that case history alone is not an adequate diagnostic tool for the diagnosis of dry eye. To avoid possible under-diagnosis, it is advised that objective clinical testing be carried out irrespective of whether the patient is symptomatic or not; this practice will facilitate a more accurate and efficient diagnosis of the presence of dry eye.

The results of the study highlight gender differences and will assist practitioners in the diagnosis and management 
of dry eye disease amongst the black and Indian population of KwaZulu-Natal in South Africa. A larger sample size incorporating all races within South Africa should be considered, and the inclusion of other dry eye tests would also be beneficial.

\section{Acknowledgements}

We thank Dr Henri Moolman (UKZN statistician) who assisted the researchers.

\section{Competing interests}

The authors declare that they have no financial or personal relationships which may have inappropriately influenced them in writing this article.

\section{Authors' contributions}

N.E.K. (University of KwaZulu-Natal) was the project leader and responsible for the project design. B.C. (University of KwaZulu-Natal), S.M. (University of KwaZulu-Natal), R.M. (University of KwaZulu-Natal), M.T.N. (University of KwaZulu-Natal), S.A.N. (University of KwaZulu-Natal) and T.A.V. (University of KwaZuluNatal) performed all the experiments, were responsible for data capturing and contributed to the manuscript writing. N.E.K. was responsible for data interpretation and manuscript writing.

\section{References}

1. Rolando $M$, Zierhut $M$. The ocular surface and tear film and their dysfunction in dry eye disease. Surv Ophthalmol. 2001;45(2):S203-210. http://dx.doi.org/10.1016/ S0039-6257(00)00203-4

2. Tiffany JM. The normal tear film. Dev Ophthalmol. 2008;41:1-20. http://dx.doi. org/10.1159/000131066

3. Kanski JJ. Clinical ophthalmology: A systematic approach. 5th edn. London: Butterworth-Heinemann; 2003.

4. Tsubota, K. Tear dynamics and dry eye. Prog Ret Eye Res. 1998;17(4):565-596. http://dx.doi.org/10.1016/S1350-9462(98)00004-4

5. Kaercher T, Bron AJ. Classification and diagnosis of dry eye. Dev Ophthalmol. 2008;41:36-53. http://dx.doi.org/10.1159/000131069

6. Dausch D, Lee S, Dausch S, Kim JC, Schwert G, Michelson W. Comparative study of treatment of the dry eye syndrome due to disturbances of the tear film lipid layer with lipid-containing tear substitutes. Klin Monatsbl Augenheilkd. 2006;223(12):974-983. http://dx.doi.org/10.1055/s-2006-927266

7. Pflugfelder SC. Differential diagnosis of dry eye conditions. Adv Dent Res. 1996;10(1):9-12. http://dx.doi.org/10.1177/08959374960100011801

8. Lemp MA, Baudouin C, Baum J, et al. The definition and classification of dry eye disease: Report of the definition and classification subcommittee of the international Dry Eye WorkShop. Ocul Surf. 2007;75:92.

9. Mertzanis P, Abetz L, Rajagopalan K, et al. The relative burden of dry eye in patient's lives: Comparisons to a U.S. normative sample. Inv Ophthalmol Vis Sci. 2005;46:46-50. http://dx.doi.org/10.1167/iovs.03-0915

10. Miljanović B, Dana R, Sullivan DA, Schaumberg DA. Impact of dry eye syndrome on vision-related quality of life. Am J Ophthalmol. 2007;143:409-415. http://dx.doi. org/10.1016/j.ajo.2006.11.060
11. Nichols KK, Mitchell GL, Zadnik K. The repeatability of clinical measurements of dry eye. Cornea. 2004;23(3):272-285. http://dx.doi.org/10.1097/00003226200404000-00010

12. Michel $M$, Sickenberger $W$, Pult $H$. The effectiveness of questionnaires in the determination of contact lens induced dry eye. Ophthal Physiol Opt. 2009;29:479486. http://dx.doi.org/10.1111/j.1475-1313.2009.00658.x

13. Narayanan S, Miller WL, Prager TC, et al. The diagnosis and characteristics of moderate dry eye in non-contact lens wearers. Eye Contact Lens. 2005;31(3):96104. http://dx.doi.org/10.1097/01.ICL.0000140907.45705.E2

14. Pult $\mathrm{H}$, Murphy PJ, Purslow $\mathrm{C}$. A novel method to predict the dry eye symptoms in new contact lens wearers. Optom Vis Sci. 2009;86(9):E1042-1050. http://dx.doi org/10.1097/OPX.0b013e3181b598cd

15. Kallarackal GU, Ansari EA, Amos N, Martin JC, Lane C, Camilleri JP. A comparative study to assess the clinical use of fluorescein meniscus time (FMT) with tear break up time (TBUT) and Schirmer's tests (ST) in the diagnosis of dry eyes. Eye. 2002;16(5):594-600. http://dx.doi.org/10.1038/sj.eye.6700177

16. Schirmer O. Studien zur physiologie und pathologie der tranenabsonderung und tranenabfuhr. Graefes Arch Clin Exp Ophthalmol. 1903;56:197-291. http://dx.doi. org/10.1007/BF01946264

17. Kashkouli MB, Pakdel F, Amani A, Asefi M, Aghai GH, Falavarjani KH. A modified Schirmer test in dry eye and normal subjects: Open versus closed eye and 1-minute versus 5-minute tests. Cornea. 2010:29(4):384-387. http://dx.doi. 1-minute versus 5-minute tests. Corn
org/10.1097/ICO.0b013e3181ba6ef3

18. Cho P, Brown B. Review of the tear break-up time and a closer look at the tear break up time of Hong Kong Chinese. Optom Vis Sci. 1993;70(1):30-38. http:// dx.doi.org/10.1097/00006324-199301000-00007

19. Savini G, Prabhawasat P, Kojima T, Grueterich M, Espana E, Goto, E. The challenge of dry eye diagnosis. Clin Ophthalmol. 2008;2(1):31-55. http://dx.doi. org/10.2147/OPTH.S1496

20. Cho P, Douthwaite W. The relation between invasive and noninvasive tear breakup time. Optom Vis Sci. 1995;72(1):17-22. http://dx.doi.org/10.1097/00006324199501000-00004

21. Gillan WDH. A small sample survey of dry eye symptoms using the ocular surface disease index. S Afr Optom. 2009;68(4):188-191.

22. Lemp MA. Advances in understanding and managing dry eye disease. Am Ophthalmol. 2008;146:350-356. http://dx.doi.org/10.1016/j.ajo.2008.05.016

23. Simpson TL, Jones LW, Fonn D. Dry eye symptoms assessed by four questionnaires. Optom Vis Sci. 2008;85(8):E692-699. http://dx.doi.org/10.1097/ OPX.0b013e318181ae36

24. Lekhanont K, Rojanaporn D, Chuck RS, Vongthongsri A. Prevalence of dry eye in Bangkok, Thailand. Cornea. 2005:25:1162-1167. http://dx.doi.org/10.1097/01. ico.0000244875.92879.1

25. Lu P, Chen X, Liu X, et al. Dry eye syndrome in early Tibetans at high altitude. Cornea. 2008;27(5): 545-551. http://dx.doi.org/10.1097/ICO.0b013e318165b1b7

26. Guo B, Lu P, Chen X, Zhang W, Chen R. Prevalence of dry eye disease in Mongolians at high altitude in China: The Henan Eye Study. Ophthalmic Epidemiol. 2010;17(4):234-241. http://dx.doi.org/10.3109/09286586.2010.498659

27. Jie Y, Xu L, Wu YY, Jonas JB. Prevalence of dry eye among adult Chinese in the Beijing Eye Study. Eye. 2009;23:688-693. http://dx.doi.org/10.1038/sj.eye.6703101

28. Lee AJ, Lee J, Saw SM, et al. Prevalence and risk factors associated with dry eye symptoms: A population based study in Indonesia. $\mathrm{Br} J$ Ophthalmol. 2002;86(12):1347-1351. http://dx.doi.org/10.1136/bjo.86.12.1347

29. Brewitt H, Sistani F. Dry eye disease: The scale of the problem. Surv Ophthalmol. 2001;45(2):S199-202. http://dx.doi.org/10.1016/S0039-6257(00)00202-2

30. Sahai A, Malik P. Dry eye: Prevalence and attributable risk factors in a hospitalbased population. Indian J Ophthalmol. 2005;53:87-91. http://dx.doi.org/ 10.4103/0301-4738.16170

31. Uchino M, Uchino Y, Dogru M, Schaumberg DA, Tsubota K. Dry eye disease in Japan: An epidemiologic study. Cornea. 2009;28(9):S31-S34. http://dx.doi. org/10.1097/ICO.0b013e3181ae9968

32. Ozdemir M, Temizdemir H. Age- and gender-related tear function changes in normal population. Eye. 2010;24:79-83. http://dx.doi.org/10.1038/eye.2009.21

33. Patel S, Farrell JC. Age-related changes in precorneal tear film stability. Optom Vis Sci. 1989;66(3):175-178. http://dx.doi.org/10.1097/00006324-19890300000008

34. Albietz J. Prevalence of dry eye subtypes in clinical optometry practice. Optom Vis Sci. 2000;77:357-363. http://dx.doi.org/10.1097/00006324-200007000-00010

35. Schein OD, Munoz B, Tielsch JM, Bandeen-Roche K, West S. Prevalence of dry eye among the elderly. Am J Ophthalmol. 1997;124:372-728. http://dx.doi. org/10.1016/S0002-9394(14)71688-5 